\title{
Minima and best approximations in constructive analysis
}

\author{
MATTHEW HENDTLASS \\ PETER SCHUSTER
}

\begin{abstract}
Working in Bishop's constructive mathematics, we first show that minima can be defined as best approximations, in such a way as to preserve the compactness of the underlying metric space when the function is uniformly continuous. Results about finding minima can therefore be carried over to the setting of finding best approximations. In particular, the implication from having at most one best approximation to having uniformly at most one best approximation is equivalent to Brouwer's fan theorem for decidable bars. We then show that for the particular case of finite-dimensional subspaces of normed spaces, these two notions do coincide. This gives us a better understanding of Bridges' proof that finite-dimensional subspaces with at most one best approximation do in fact have one. As a complement we briefly review how the case of best approximations to a convex subset of a uniformly convex normed space fits into the unique existence paradigm.
\end{abstract}

2000 Mathematics Subject Classification 03F60 (primary); 41A50,41A52 (secondary)

Keywords: minima,best approximations, finite-dimensional subspace, uniformly convex, fan theorem, constructive

\section{Introduction}

The present paper is set in the realm of Bishop's constructive mathematics (BISH) ${ }^{1}$. Bishop's constructive framework is distinguished from classical - that is traditionalmathematics by a strict adherence to intuitionistic logic. This allows us to view BISH as a generalisation of classical mathematics [43]: adding the law of excluded middle recovers classical logic.

To start with we recall some background material. Let $(X, d)$ be a metric space. Any such $X$ is compact if it is totally bounded and complete, where being complete means

\footnotetext{
${ }^{1}$ We refer to $[13,14,21,24]$ for a development of constructive analysis in BISH, and to $[1,16,27,42]$ for constructive alternatives to ZFC.
} 
that every Cauchy sequence converges. Moreover, a subset $S$ of $X$ is located if for each $z \in X$ the distance

$$
\operatorname{dist}(z, S)=\inf _{x \in S} d(x, z)
$$

from $x$ to $S$ exists. Any totally bounded subset of a metric space is located.

Continuity is a thorny issue in constructive mathematics ${ }^{2}$. Since the three major models of BISH - classical mathematics, Brouwer's intuitionistic mathematics, and the Russian school of recursive mathematics ${ }^{3}$ - all disagree on which continuity principles hold, we must be very careful; in particular, in recursive mathematics there is a continuous function on the unit interval which is not uniformly continuous. In [13], Bishop elegantly sidesteps this problem by including the uniform continuity theorem in the definition of continuity: $f$ is a Bishop-continuous function if $S$ is locally totally bounded and $f$ is uniformly continuous on each compact subset of $S$. Although this solution allowed Bishop and his followers to develop a significant body of results, we will only ever need either pointwise or uniform continuity in this paper; when we refer to a function as continuous we mean that it is pointwise continuous.

In the following we loosely follow [50]. Let $f$ be a continuous real-valued function on (a subset $S$ of) $X$. For the sake of a simpler presentation we assume that $f \geqslant 0$. The conditional existence problem

$$
\text { If the infimum of } f \text { on } S \text { is } 0 \text {, can we construct } \xi \in S \text { such that } f(\xi)=0 \text { ? }
$$

incorporates, with $f: S \rightarrow \mathbf{R}$ defined by

$$
f(x)=d(x, z)-\operatorname{dist}(z, S),
$$

the search for best approximations to a point $z$ of $X$ in a located subset $S$ of $X$. That is to say, a best approximation to $z$ in $S$ is nothing but a point of $S$ at which the uniformly continuous function $d(\cdot, z): S \rightarrow \mathbb{R}$ attains its infimum $\operatorname{dist}(z, S)$. This, however, does not mean that a best approximation can always be constructed, even if $S$ is compact-see below.

A natural first step towards solving the general problem is to construct-using countable choice-a sequence $\left(x_{n}\right)$ in $S$ such that

$$
f\left(x_{n}\right)<1 / n
$$

\footnotetext{
${ }^{2}$ See, for instance, [48] for more on continuity in constructive analysis.

${ }^{3}$ See [21] for an introduction to the intuitionistic and recursive schools of mathematics and their relationship to classical mathematics and Bishop's constructive mathematics.
} 
for each $n \geqslant 1$, which is indeed possible whenever $f$ restricted to $S$ has infimum 0 . If $S$ is compact, working classically we could then, using the Bolzano-Weierstrass theorem

BWT Every sequence in a compact metric space has a convergent subsequence,

select a convergent subsequence of $\left(x_{n}\right)$, whose limit $\xi$ would moreover satisfy $f(\xi)=$ 0 . In fact, the minimum theorem

MIN Every uniformly continuous function on a compact metric space has a minimum,

which would solve our problem with a single stroke of the pen, is a simple consequence of BWT. However, MIN, and therefore BWT, is essentially non-constructive [28]. ${ }^{4}$

The non-constructive character of MIN notwithstanding, one can show constructively that

$$
\inf f=\inf _{x \in X} f(x)
$$

exists whenever $f: X \rightarrow \mathbb{R}$ is uniformly continuous, and $X$ is totally bounded [21, Chapter 2, Theorem 4.5]. So if we restrict ourselves to continuous functions on compact spaces our problem becomes

When does a uniformly continuous function on a compact metric space attain its infimum?

We now drop the assumption that $f \geqslant 0$, but suppose that inf $f$ can be computed. A function $f: X \rightarrow \mathbb{R}$ is said to have uniformly at most one minimum [49] if

$$
\forall_{\varepsilon>0} \exists_{\delta>0} \forall_{x, y \in X}(\max \{f(x), f(y)\}<\inf f+\delta \Rightarrow d(x, y)<\varepsilon)
$$

or equivalently

$$
\forall_{\varepsilon>0} \exists_{\delta>0} \forall_{x, y \in X}(d(x, y) \geqslant \varepsilon \Rightarrow f(x) \geqslant \inf f+\delta \vee f(y) \geqslant \inf f+\delta) .
$$

If $f$ has uniformly at most one minimum, then $f$ has at most one minimum [9]:

$$
\forall_{\varepsilon>0} \forall_{x, y \in X} \exists_{\delta>0}(d(x, y) \geqslant \varepsilon \Rightarrow f(x) \geqslant \inf f+\delta \vee f(y) \geqslant \inf f+\delta)
$$

or equivalently

$$
\forall_{x, y \in X}(x \neq y \Rightarrow f(x)>\inf f \vee f(y)>\inf f) .
$$

If $f$ has at most one minimum, then any point—if one exists—at which $f$ attains its infimum is unique; that is, if $x, y \in X$ are such that $f(x)$ and $f(y)$ are both equal to $\inf f$, then $x=y$.

\footnotetext{
${ }^{4}$ An outline of this, including references, can be found e.g. in [49].
} 
Now let $X$ be complete. As recalled in [49], if $f$ has uniformly at most one minimum, then $f$ actually has a minimum: that is, $f$ attains its infimum. ${ }^{5}$ To prove this, one can construct, with countable choice as in the classical argument discussed before, a sequence $\left(x_{n}\right)$ in $X$ such that

$$
f\left(x_{n}\right)<1 / n
$$

for each $n \geqslant 1$. Now the additional hypothesis can be used that $f$ has uniformly at most one minimum, to show that $\left(x_{n}\right)$ is a Cauchy sequence. Since $X$ is complete, $\left(x_{n}\right)$ has a limit $\xi$ in $X$, for which clearly $f(\xi)=\inf f$.

Hence if $X$ is complete and $f$ has uniformly at most one minimum, then one can construct the a fortiori unique minimum of $f$. Even if $X$ fails to be complete, the given data can be converted into an element of the completion of $X$ : namely, into the Cauchy sequence $\left(x_{n}\right)$ in $X$. This observation has suggested a way [50] ${ }^{6}$ to get by in Bishop-style constructive mathematics without countable choice as proposed by Richman [44, 45], where completions are defined without sequences.

Being essentially folklore, this problem and its solution have some history. The first known occurrence is in Theorem 4 of Lifschitz's [38] - however, there is no proof in the English translation of the Russian original from 1971. Kreinovich [37] also refers to a related metatheorem by M.G. Gelfond from 1972. The work of Bridges in the 1980s was centred around best approximations; see [19] for an overview and for references. To our knowledge, the next occurrence of this argument is Problem 10, ascribed to Aczel, in Chapter 2 of Bridges and Richman's [21]. Uniform uniqueness in the form of moduli of uniqueness was then used intensively by Kohlenbach from the early 1990s [34], first also in the context of best approximations. ${ }^{7}$

Recently unique existence has further occurred in work by Brattka [15] in computable analysis à la Weihrauch [56], and by Taylor [53] within his abstract Stone duality, see also [2]. In both cases non-uniform uniqueness suffices because versions of the Heine-Borel theorem are available. As noticed in [49], the statement

UAM If a uniformly continuous function on a compact metric space has at most one minimum, then it has uniformly at most one minimum

is indeed equivalent ${ }^{8}$ to Brouwer's fan theorem for decidable bars, the paradigmatic version of the Heine-Borel theorem [21], which can be put as 9

\footnotetext{
${ }^{5}$ This is analogous to the rule from [34, Theorem 4.4].

${ }^{6}$ Diener and the second author [26] have applied this to the implicit functions theorem.

${ }^{7}$ We refer to Kohlenbach and Oliva's survey [35] and to Kohlenbach's book [36] for more recent developments.

${ }^{8}$ One half of this equivalence corresponds to the rule from [34, Theorem 4.3].

${ }^{9}$ See, for instance, $[11,32]$. FT is the classical contrapositive of the Weak König Lemma.
} 
FT Every decidable binary tree without an infinite path is finite.

This classification in the spirit of constructive reverse mathematics-as practised by Ishihara $[29,30,31]$ and others ${ }^{10}$ — sharpens an earlier result the second author has obtained jointly with Berger and Bridges [9]; see also [10, 32, 33, 52].

In the first part of the present note the classification of UAM is carried over from the context of minima to the more specific context of best approximations that was considered much earlier. In the second part we have a closer look at a prime case of the latter where a best approximation of a point in a normed space is sought in a finitedimensional subspace. We conclude with a brief discussion of best approximations in a convex subset to a point in a uniformly convex normed space.

\section{Minima and best approximations}

We first show how minima can be expressed as best approximations. Let $X$ be a metric space with metric $d$, let $S$ be a located subset of $X$, and let $x$ be a point of $X$. A best approximation to $x$ in $S$ is a minimum point of the function $f: S \rightarrow \mathbf{R}$ defined by

$$
f(s)=d(x, s) .
$$

Moreover, $x$ has (uniformly) at most one best approximation in $S$ if $f$ has (uniformly) at most one minimum in $S$. The following theorem shows conversely that minima of a function on a metric space into $\mathbf{R}$ which is bounded below can be seen as best approximations.

Theorem 1 Let $f$ be a function from a metric space $(X, d)$ into $\mathbf{R}$ which is bounded below. By * we denote a point that is not in $X$. There exists a metric $\rho$ on $X \cup\{*\}$ such that

$$
f(x)=\rho(*, x)
$$

for every $x \in X$; in particular, the minima of $f$ in $X$ are exactly the best approximations to $*$ in $X$. Moreover, inf $f$ can be computed if and only if $\operatorname{dist}_{\rho}(*, X)$ can be computed, in which case

$$
\inf f=\operatorname{dist}_{\rho}(*, X) .
$$

If, in addition, $(X, d)$ is bounded, then $f$ has (uniformly) at most one minimum in $(X, d)$ precisely when $*$ has (uniformly) at most one best approximation in $(X, \rho)$. Finally, if $(X, d)$ is compact and $f$ is uniformly continuous, then $(X, \rho)$ is compact.

\footnotetext{
${ }^{10}$ See, for instance, $[3,4,5,6,7,8,11,12,20,25,39,40,41,54,55]$
} 
Proof Let $f$ be a function from $(X, d)$ into $\mathbf{R}$ which is bounded below, let $*$ be some point not in $X$, and let $X^{\prime}=X \cup\{*\}$. We first replace $d$ by the metric $d^{\prime}=d /(1+d)$ on $X$, with respect to which $X$ is bounded with diameter no greater than 1 , and define $g: X \rightarrow \mathbf{R}$ by

$$
g(x)=f(x)-1 / 2
$$

since $f$ is bounded below, we may assume that $g(x) \geqslant 0$ for all $x$. We next extend $d^{\prime}$ to be a metric on $X^{\prime}$ by setting $d^{\prime}(*, x)=1 / 2$ for all $x \in X$; the only nontrivial property to verify is the following instance of the triangle inequality: for all $x, y \in X$ we have

$$
d^{\prime}(x, y) \leqslant 1=d^{\prime}(x, *)+d^{\prime}(*, y),
$$

because the diameter of $\left(X, d^{\prime}\right)$ is bounded by 1 . We finally extend $g$ to $X^{\prime}$ by setting $g(*)=0$, and define $\rho: X^{\prime} \times X^{\prime} \rightarrow \mathbf{R}$ by

$$
\rho(x, y)=d^{\prime}(x, y)+|g(x)-g(y)|,
$$

for which clearly $d^{\prime} \leqslant \rho$. It is easy to see that $(x, y) \mapsto|g(x)-g(y)|$ is a pseudo-metric on $X^{\prime}$; whence $\left(X^{\prime}, \rho\right)$ is a metric space. Moreover,

$$
\begin{aligned}
\rho(*, x) & =d^{\prime}(*, x)+|g(x)-g(*)| \\
& =1 / 2+|g(x)| \\
& =1 / 2+g(x) \\
& =f(x)
\end{aligned}
$$

for every $x$. In particular, each minimum of $f$ corresponds to a best approximation to * in the subset $X$ of $\left(X^{\prime}, \rho\right)$, and $\operatorname{dist}_{\rho}(*, X)$ can be computed precisely when $\inf f$ can be computed, in which case they are equal.

We now assume that $(X, d)$ is bounded, and show that $f$ has (uniformly) at most one minimum in $(X, d)$ if and only if $*$ has (uniformly) at most one best approximation in $(X, \rho)$. Since $X$ is bounded, $d$ and $d^{\prime}$ are similar metrics (i.e. each of them is bounded by the other up to a nonnegative multiplicative constant), from which-together with $d^{\prime} \leqslant \rho$ - the "if" part of the desired equivalence follows.

As for the "only if" part, since the non-uniform case is completely analogous, we may focus on the uniform case. Let $\varepsilon>0$ be given, and take $\delta>0$ as in " $f$ has uniformly at most one minimum in $(X, d)$ " such that $\delta \leqslant \varepsilon / 3$. Now if $x, y \in X$ are such that $f(x)$ and $f(y)$ are both smaller than $\inf f+\delta$, then

$$
\begin{aligned}
\rho(x, y) & =d^{\prime}(x, y)+|g(x)-g(y)| \\
& \leqslant d(x, y)+|f(x)-f(y)| \\
& <\varepsilon / 3+2 \delta \\
& \leqslant \varepsilon .
\end{aligned}
$$


To conclude we show that

- if $(X, d)$ is complete and bounded, and $f$ is continuous, then $(X, \rho)$ is complete;

- if $(X, d)$ is totally bounded and $f$ is uniformly continuous, then $(X, \rho)$ is totally bounded.

Suppose first that $(X, d)$ is complete and bounded, and that $f$, and hence $g$, is continuous. Let $\left(x_{n}\right)$ be a Cauchy sequence in $(X, \rho)$; it then also is a Cauchy sequence in $\left(X, d^{\prime}\right)$, because $d^{\prime} \leqslant \rho$. But, since $X$ is bounded, $d$ and $d^{\prime}$ are similar metrics as above, so $\left(X, d^{\prime}\right)$ is complete; whence there exists $x \in X$ such that $x_{n} \rightarrow x$ in $\left(X, d^{\prime}\right)$ as $n \rightarrow \infty$. Since $g$ is continuous (with respect to $d$ and thus also with respect to $d^{\prime}$ ), we have

$$
\rho\left(x, x_{n}\right)=d^{\prime}\left(x, x_{n}\right)+\left|g(x)-g\left(x_{n}\right)\right| \rightarrow 0
$$

as $n \rightarrow \infty$. Thus $x_{n} \rightarrow x$ in $(X, \rho)$ as $n \rightarrow \infty$, so $(X, \rho)$ is complete.

Suppose next that $(X, d)$ is totally bounded and that $f$, and hence $g$, is uniformly continuous. Again $d$ and $d^{\prime}$ are similar metrics, so $\left(X, d^{\prime}\right)$ is totally bounded. Fix $\varepsilon>0$ and let $\delta \in(0, \varepsilon / 2)$ be such that for all $x, y \in X$, if $\rho(x, y)<\delta$, then $|g(x)-g(y)|<\varepsilon / 2$. Let $\left\{x_{1}, \ldots, x_{n}\right\}$ be a $\delta$-approximation to $\left(X, d^{\prime}\right)$. Let $x \in X$, and pick $1 \leqslant i \leqslant n$ such that $d^{\prime}\left(x, x_{i}\right)<\delta$. Then

$$
\begin{aligned}
\rho\left(x, x_{i}\right) & =d^{\prime}\left(x, x_{i}\right)+\left|g(x)-g\left(x_{i}\right)\right| \\
& <\varepsilon / 2+\varepsilon / 2 \\
& =\varepsilon .
\end{aligned}
$$

Hence $\left\{x_{1}, \ldots, x_{n}\right\}$ is an $\varepsilon$-approximation to $(X, \rho)$.

From what has been said before, it is immediate that if a subset $S$ of a metric space $X$ is complete and has uniformly at most one best approximation to a point $x$ in $X$, then there is a (necessarily unique) best approximation to $x$ in $S$.

We can now classify the counterpart of UAM for best approximations:

UAB If a compact subset $S$ of a metric space $X$ has at most one best approximation to a point $x$ of $X$, then $S$ has uniformly at most one best approximation to $x$.

\section{Corollary 2 UAB is equivalent to FT.}

Proof It is clear that UAM implies UAB, whereas the converse implication follows from Theorem 1. Since UAM is equivalent to FT [49], so is UAB. 


\section{Finite-dimensional subspaces}

We now turn our attention to a fundamental result in constructive approximation theory [17]. Let $X$ be a normed space, let $x \in X$, and let $S$ be a located subset of $X$. It is clear that $S$ has uniformly at most one best approximation to $x$ precisely when for each $\varepsilon>0$ there exists $\delta>0$ such that, for all $y, y^{\prime} \in S$, if

$$
\max \left\{\|x-y\|,\left\|x-y^{\prime}\right\|\right\}<\operatorname{dist}(x, S)+\delta,
$$

then $\left\|y-y^{\prime}\right\|<\varepsilon$, whereas $S$ has at most one best approximation to $x$ if and only if

$$
\max \left\{\|x-y\|,\left\|x-y^{\prime}\right\|\right\}>\operatorname{dist}(x, S)
$$

for all distinct $y, y^{\prime} \in S$. While the latter notion of (non-uniform) uniqueness was coined in [17], a located subset $S$ was called quasi-proximinal in [18] if every $x \in X$ with at most one best approximation in $S$ has a, necessarily unique, best approximation in $S$.

Finitely many vectors $e_{1}, \ldots, e_{n}$ in $X$ are linearly independent if $\lambda_{1} e_{1}+\ldots+\lambda_{n} e_{n} \neq 0$ whenever $\left|\lambda_{1}\right|+\ldots+\left|\lambda_{n}\right|>0$. A subspace $E$ of $X$ is finite-dimensional [17] if there exist linearly independent vectors $e_{1}, \ldots, e_{n} \in E$ and bounded linear functionals $u_{i}: E \rightarrow \mathbf{R}(1 \leqslant i \leqslant n)$, the component functions, such that

$$
x=\sum_{i=1}^{n} u_{i}(x) e_{i}
$$

for all $x$ in $E$; in this case $n$ is called the dimension of $E$, which of course is uniquely determined by $E$. Any finite-dimensional subspace $E$ of $X$ is located [17, Proposition 2.1] and complete [14, p. 307]; in particular, $E$ is a closed subset of $X{ }^{11}$ We denote by $\mathbf{R} e$ the one dimensional subspace spanned by a nonzero element $e$ of $X$. A subset $S$ of $X$ is said to be convex if $t y+(1-t) y^{\prime} \in S$ whenever $y, y^{\prime} \in S$ and $t \in[0,1]$.

Theorem 3 Each finite-dimensional subspace of a normed space is quasi-proximinal.

In the following we give a dissection of Bridges' proof of Theorem 3 above; see Theorem 2.2 of [17], the (only) Theorem of [18], and Chapter 7, Theorem 2.12 of [14]. We do this with three goals in mind:

- to recast the proof in terms of uniform uniqueness;

- to elucidate the structure of the proof, and hence the structure of the inherent algorithm;

\footnotetext{
${ }^{11}$ In [14] locatedness and closedness are included into the definition of a subspace.
} 
— to further illustrate the relationship between minima and best approximations.

Let $X$ be a normed space, let $x \in X$, let $E$ be a finite-dimensional subspace of $X$, and let $S$ be a located convex subset of $E$. For $\delta>0$ we define

$$
S_{\delta}=\{e \in S:\|x-e\|<\operatorname{dist}(x, S)+\delta\} .
$$

Then for each positive $\delta, S_{\delta}$ is inhabited and-as the intersection of the convex set $S$ with an open ball-bounded and convex. If $\delta_{1}>\delta_{2}>0$, then $S_{\delta_{2}} \subset S_{\delta_{1}}$. We can rewrite the condition that $S$ has uniformly at most one best approximation to $x$ as

$$
\forall_{\varepsilon>0} \exists_{\delta>0} \forall_{y, y^{\prime} \in S_{\delta}}\left(\left\|y-y^{\prime}\right\|<\varepsilon\right) .
$$

We begin with, a slight extension of, the 1-dimensional case of Theorem 3, where the presence of a total ordering makes life much easier.

Lemma 4 Let $X$ be a normed space, let $x, e \in X$ with $e \neq 0$, and let $S$ be a located convex subset of $\mathbf{R} e$. If $x$ has at most one best approximation in $S$, then $x$ has uniformly at most one best approximation in $S$.

Proof Fix $\varepsilon>0$, replace $e$ by $e /\|e\|$, and let $t_{1}<\cdots<t_{n}$ be real numbers such that $\left\{t_{1} e, \ldots, t_{n} e\right\}$ is an $\varepsilon / 5$-approximation to $S$. If $n \in\{1,2\}$, then, since $\|e\|=1$, the diameter of $S_{1}$ is less than $\varepsilon$ and we need only set $\delta=1$; therefore we may assume that $n>2$. Since $x$ has at most one best approximation in $S$, there exists $\delta>0$ and $i_{0} \in\{1, \ldots, n\}$ such that $\left\|x-t_{i} e\right\|>\delta$ for all $i \neq i_{0}$. Let $t \in S_{\delta}$; then $t \neq t_{i}$ for all $i \neq i_{0}$. Given that $S_{\delta}$ is nonempty and convex, we must have that $\left\|t e-t_{1} e\right\|<\varepsilon / 5$, $\left\|t e-t_{n} e\right\|<\varepsilon / 5$, or there exists $j \neq i_{0}$ such that $S_{\delta} \subset\left(t_{j}, t_{j+2}\right) e$. In the first case, $S_{\delta} \subset\left(-\infty, t_{3}\right) e \cap S$ which has diameter less than $\varepsilon$ since $\|e\|=1$ and by our choice of $t_{1}, \ldots, t_{n}$; similarly, in the second case $S_{\delta} \subset\left(t_{n-2}, \infty\right) e \cap S$, so $\operatorname{diam}(S)<\varepsilon$. In the final case $\operatorname{diam}(S)<2 \varepsilon / 5$. Hence in all cases, for all $y, y^{\prime} \in S_{\delta},\left\|y-y^{\prime}\right\|<\varepsilon$.

Corollary 5 Let $X$ be a normed space, let $x, e \in X$ with $e \neq 0$, and let $S$ be a closed located convex subset of $\mathbf{R} e$. If $x$ has at most one best approximation in $\mathbf{R} e$, then $x$ has a best approximation in $S$.

Extending this to encompass Bridges' main lemma [18] seems to require countable choice, at least in the weak form given in [23] that is classically valid without any choice.

Lemma 6 Let $X$ be a normed space, let $x, e \in X$ with $e \neq 0$, let $S$ be a closed located convex subset of $\mathbf{R} e$, and let $d \in \mathbf{R}$ be such that

$$
\max \left\{\|x-s\|,\left\|x-s^{\prime}\right\|\right\}>d
$$


for all distinct $s, s^{\prime} \in S$. Then there exists $\tau \in \mathbf{R}$ such that $\tau e \in S$ and if $\|x-\tau e\|>d$, then $\operatorname{dist}(x, S)>d$.

Proof We first note that $d \leqslant \operatorname{dist}(x, S)$. Let $\left(\lambda_{n}\right)$ be an increasing binary sequence such that

$$
\begin{aligned}
& \lambda_{n}=0 \Rightarrow \operatorname{dist}(x, S)-d<1 / n, \\
& \lambda_{n}=1 \Rightarrow \operatorname{dist}(x, S)-d>1 /(n+1),
\end{aligned}
$$

and, using Lemma 4, construct a sequence $\left(\delta_{n}\right)$ of positive real numbers such that $\operatorname{diam}\left(S_{\delta_{n}}\right)<1 /(n+1)$ for each $n$. We construct a Cauchy sequence $\left(t_{n}\right)$ in $\mathbf{R}$ as follows. If $\lambda_{n}=0$, pick $t \in S_{\delta_{n}}$ and set $t_{n}=t$; if $\lambda_{n}=1$ set $t_{n}=t_{n-1}$. Let $\tau$ be the limit of $\left(t_{n}\right)$ in $\mathbf{R}$. Since $S$ is closed, we have $\tau e \in S$. Suppose that $\|x-\tau e\|>d$ and let $N>0$ be such that

$$
\|x-\tau e\|>d+2 / N .
$$

If $\lambda_{N}=0$, then $\|x-\tau e\|<\operatorname{dist}(x, S)+1 / N$ and $\operatorname{dist}(x, S)-d<1 / N$, so

$$
\|x-\tau e\|<\operatorname{dist}(x, S)+1 / N<d+2 / N .
$$

This contradiction ensures that $\lambda_{N}=1$; whence $\operatorname{dist}(x, S)>d$.

The particular case $d=\operatorname{dist}(x, \mathbf{R} e)$ of Lemma 6 says that if $S$ has at most one best approximation to $x$, then it does have a-necessarily unique — best approximation.

In [17], Bridges proceeds to prove Theorem 3 by an induction on the dimension of the finite-dimensional subspace. The next proposition isolates this induction. (The previous extension to closed located convex subsets of the finite-dimensional subspaces breaks down here; a counterexample can be constructed by extending that, in [17], of a linear subspace without a best approximation.) We need a few more definitions, and a lemma from [24]. Let $E$ be a closed located subspace of a normed space $X$. We define a semi-norm $\|\cdot\|_{X / E}$ on $X$ by

$$
\|x\|_{X / E}=\rho(x, E)
$$

With the inequality given by

$$
x \neq y \Leftrightarrow\|x-y\|_{X / E} \neq 0
$$

$\left(X,\|\cdot\|_{X / E}\right)$ becomes a normed space called the quotient space of $X$ by $E$, written $X / E$; we read $X / E$ as $X$ modulo $E$. The following is Lemma 4.2.2 of [24]: 
Lemma 7 Let $\left\{e_{1}, \ldots, e_{n}\right\}$ be a basis for an $n$-dimensional subspace $V$ of a normed space $X$, let $1 \leqslant m<n$, and let $W$ be the subspace of $X$ with basis $\left\{e_{1}, \ldots, e_{m}\right\}$. Then the span of $\left\{e_{m+1}, \ldots, e_{n}\right\}$ is an $(n-m)$-dimensional subspace of the quotient space $X / W$.

Proposition 8 Let $X$ be a normed space, let $E$ be a finite-dimensional subspace of $X$, and let $x \in X$ be such that $x$ has at most one best approximation in $E$. Then for each finite-dimensional subspace $E^{\prime}$ of $E, x$ has at most one best approximation in $E / E^{\prime}$ (viewed as a subspace of $X / E^{\prime}$ ).

Proof We proceed by induction on the dimension of $E$. If $E=\mathbf{R} e$, then the result is trivial. Now suppose that we have proved the result for subspaces with dimension $n-1$, let $\left\{e_{1}, \ldots, e_{n}\right\}$ be a basis for $E$, and let $E^{\prime}$ be a finite-dimensional linear subspace of $E$. Without loss of generality $e_{1} \in E^{\prime}$.

Let $v_{1}, v_{2} \in E$ be such that $\left\|v_{1}-v_{2}\right\|_{X / \mathbf{R} e_{1}}>0$. If $t, t^{\prime} \in \mathbf{R}$ with $t \neq t^{\prime}$, then for $i=1,2$

$$
\left\|\left(v_{i}-t e_{1}\right)-\left(v_{i}-t^{\prime} e_{1}\right)\right\|=\left|t-t^{\prime}\right|\left\|e_{1}\right\| \neq 0
$$

so

$$
\max \left\{\left\|x-\left(v_{i}-t e_{1}\right)\right\|,\left\|x-\left(v_{i}-t^{\prime} e_{1}\right)\right\|\right\}>\operatorname{dist}(x, E) .
$$

Applying Lemma 6 with $x=x-v_{i}, e=e_{1}$, and $d=\operatorname{dist}(x, E)$ produces $\tau_{i}$ such that if $\left\|x-\left(v_{i}-\tau_{i} e_{1}\right)\right\|>\operatorname{dist}(x, E)$, then $\operatorname{dist}\left(x-v_{i}, \mathbf{R} e_{1}\right)>\operatorname{dist}(x, E)$.

Since $x$ has at most one best approximation in $E$, either

$$
\left\|x-v_{1}-\tau_{1} e_{1}\right\|>\operatorname{dist}(x, E) \text { or }\left\|x-v_{2}-\tau_{2} e_{1}\right\|>\operatorname{dist}(x, E) .
$$

Hence

$$
\max \left\{\operatorname{dist}\left(x-v_{1}, \mathbf{R} e_{1}\right), \operatorname{dist}\left(x-v_{2}, \mathbf{R} e_{1}\right)\right\}>\operatorname{dist}(x, E),
$$

so $x$ has at most one best approximation in $E$ modulo $\mathbf{R} e_{1}$. Since, by Lemma 7, $E / \mathbf{R} e_{1}$ is an $(n-1)$-dimensional subspace of $X / \mathbf{R} e_{1}$, it follows from our induction hypothesis that $x$ has at most one best approximation in $E$ modulo $\mathbf{R} e_{1}$ modulo $E^{\prime}$. Since $X / E^{\prime}=\left(X / \mathbf{R} e_{1}\right) / E^{\prime}$, this completes the induction.

We can now give the uniform uniqueness version of Theorem 3, from which that theorem follows.

Theorem 9 Let $X$ be a normed space, let $E$ be a finite-dimensional subspace of $X$, and let $x \in X$ be such that $x$ has at most one best approximation in $E$. Then $x$ has uniformly at most one best approximation in $E$. 
Proof Let $\varepsilon>0$ and let $e_{1}, \ldots, e_{n}$ be a basis for $E$ with component functions $u_{1}, \ldots, u_{n}$. Since all norms on a finite-dimensional subspace are equivalent (Corollary 4.1.9 of [24]), it suffices to show that there exists $\delta>0$ such that

$$
\left|u_{i}(y)-u_{i}\left(y^{\prime}\right)\right|<\varepsilon
$$

for all $y, y^{\prime} \in S_{\delta}$ and each $i \in\{1, \ldots, n\}$.

For each $i \in\{1, \ldots, n\}$ let

$$
E_{i}=\operatorname{span}\left\{e_{j}: 1 \leqslant j \leqslant n, j \neq i\right\} .
$$

By Proposition 8, $x$ has at most one best approximation in $E$ modulo $E_{i}$ for each $i$. Using Lemma 4 , construct $\delta_{1}, \ldots, \delta_{n}$ such that if

$$
\left\|x-t e_{i}\right\|_{X / E_{i}}<\operatorname{dist}_{X / E_{i}}\left(x, \mathbf{R} e_{i}\right)+\delta_{i},
$$

then $\left|t-t^{\prime}\right|<\varepsilon$. It remains to set $\delta=\min \left\{\delta_{i}: 1 \leqslant i \leqslant n\right\}$.

\section{Convex subsets}

We finally review the case of best approximations to a convex set in a uniformly convex normed space. In all the sources referred to in this section the following definition, a uniform-quantitative variant of the one of a strictly convex normed space, has been put in one way or the other: a normed space $X$ is uniformly convex if for all $\varepsilon>0$ there exists $\delta>0$ such that

$$
\|(x+y) / 2\|>1-\delta \Rightarrow\|x-y\|<\varepsilon
$$

for all $x, y \in X$ with $\|x\|=\|y\|=1$. (In [36] the dependence of $\delta$ on $\varepsilon$ is expressed as a modulus of uniform convexity.)

With Chapter 9, Exercise 5 of [13] and again with Chapter 7, Exercise 11 of [14], which in this section will be referred to as Bishop's exercise, it is claimed that the following statement has a constructive proof: if $S$ is a closed located convex subset of a uniformly convex Banach space $X$, then each point in $X$ has a unique best approximation in $S$. (Proposition 17.5 of [36] says the same, with a modulus of uniform convexity for $X$.) Note that there is no need to suppose that the uniformly convex normed space $X$ be complete: it suffices to assume that the convex located subset $S$ is complete.

Since finite-dimensional (linear) subspaces are complete located convex subsets (see above), Theorem 3.1 of [17] is a special case of Bishop's exercise. This Theorem 3.1 says that if $E$ is a finite-dimensional subspace of a uniformly convex Banach space $X$, 
then each point in $X$ has a, necessarily unique, best approximation in $E$. It was proved by what then was a new technique involving uniqueness; more precisely Theorem 3.1 was reduced to Theorem 2.2 of [17], a forerunner of the quasi-proximinality of finite-dimensional subspaces of arbitrary normed spaces (see Theorem 3 above). An inspection of the very proof of this Theorem 3.1 reveals that each point in a uniformly convex normed space $X$ has indeed uniformly at most one best approximation in any given finite-dimensional subspace $E$ of $X$.

The content of Theorem 6 of [22] is that if $E$ is a complete located subspace of a uniformly convex normed space $X$, then each point of $X$ has a unique best approximation in $E$. Inasmuch as $E$ need not be finite-dimensional, this clearly generalises Theorem 3.1 of [17].

When going through the proof of Theorem 6 of [22] one will notice that $E$ may even be a convex subset rather than a (linear) subspace; whence one might think that this theorem is nothing but a remake of Bishop's exercise. The point of Theorem 6 of [22] is, however, that its proof is done without countable choice, again with a concept of a completion that is free of sequences; on the other hand it is clear that in the intended proof of Bishop's exercise a sequence would have to be chosen to approximate the best approximation.

Last but not least, by looking at the proof of Theorem 6 of [22] together with its Lemma 5 we can exhibit the essence of Bishop's exercise: if $S$ is a convex located subset of a uniformly convex normed space $X$, then each point in $X$ has uniformly at most one best approximation in $S$. (This is Proposition 17.4 of [36], again with moduli of uniform convexity and uniqueness.)

\section{References}

[1] P.H.G. Aczel and M. Rathjen, Notes on Constructive Set Theory, Report No. 40, Institut Mittag-Leffler, Royal Swedish Academy of Sciences, 2001; http://www.ml.kva.se/preprints/archive/2000-2001/2000-2001-40.pdf.

[2] A. Bauer, and P. Taylor, The Dedekind reals in abstract Stone duality, Math. Structures Comput. Sci. 19 (2009); 757-838; doi:10.1017/S0960129509007695.

[3] J. Berger, The weak König lemma and uniform continuity, J. Symbolic Logic 73 (2008), 933-939; doi:10.2178/jsl/1230396756.

[4] J. Berger, A decomposition of Brouwer's fan theorem, J. Log. Anal. 1 (2009), Paper 6; doi:10.4115/jla.2009.1.6. 
[5] J. Berger and D.S. Bridges, A bizarre property equivalent to the $\Pi_{1}^{0}$-fan theorem, Log. J. IGPL 14 (2006), 867-871; doi:10.1093/jigpal/jzl026.

[6] J. Berger and D.S. Bridges, A fan-theoretic equivalent of the antithesis of Specker's theorem, Indag. Math. (N.S.) 18 (2007), 195-202; doi:10.1016/S0019-3577(07)000122.

[7] J. Berger and D.S. Bridges, The fan theorem and positive-valued uniformly continuous functions on compact intervals, New Zealand J. Math. 38 (2008), 129-135.

[8] J. Berger and D.S. Bridges, The anti-Specker property, a Heine-Borel property, and uniform continuity, Arch. Math. Logic 46 (2008), 583-592; doi:10.1007/s00153-0070063-1.

[9] J. Berger, D.S. Bridges, and P. Schuster, The fan theorem and unique existence of maxima, J. Symbolic Logic 71 (2006), 713-720; doi:10.2178/js1/1146620167.

[10] J. Berger and H. Ishihara, Brouwer's fan theorem and unique existence in constructive analysis, Math. Log. Quart. 51 (2005), 369-373; doi:10.1002/malq.200410038.

[11] J. Berger and P. Schuster, Classifying Dini's theorem, Notre Dame J. Formal Logic 47 (2006), 253-262; doi:10.1305/ndjfl/1153858650.

[12] J. Berger and P. Schuster, Dini's theorem in the light of reverse mathematics, In: S. Lindström, E. Palmgren, K. Segerberg, V. Stoltenberg-Hansen, eds., Logicism, Intuitionism, and Formalism - What has become of them? Workshop on the Philosophy of Mathematics, Uppsala, Sweden, August 27-29, 2004. Springer, Dordrecht. Synth. Libr. 341 (2009), 153-166; doi:10.1007/978-1-4020-8926-8_7.

[13] E. Bishop, Foundations of Constructive Analysis, McGraw-Hill, New York, 1967.

[14] E. Bishop and D.S. Bridges, Constructive Analysis, Springer, Berlin etc., 1985.

[15] V. Brattka, Borel complexity and computability of the Hahn-Banach theorem, Arch. Math. Logic 46 (2008), 547-564; doi:10.1007/s00153-007-0057-z.

[16] D.S. Bridges, A constructive Morse theory of sets, Mathematical Logic and its Applications, Plenum Publishing Corp., New York, 61-79, 1987.

[17] D.S. Bridges, On the foundations of best approximation theory, J. Approx. Theory 28 (1980), 273-292; doi:10.1016/0021-9045(80)90081-7.

[18] D.S. Bridges, A constructive proximinality property of finite-dimensional linear subspaces, Rocky Mountain J. Math. 11 (1981), 491-497.

[19] D.S. Bridges, Recent progress in constructive approximation theory, The L.E.J. Brouwer Centenary Symposium, North-Holland, Amsterdam (1982), 41-50; doi:10.1016/S0049-237X(09)70121-1.

[20] D. Bridges and H. Diener, The pseudocompactness of $[0,1]$ is equivalent to the uniform continuity theorem, J. Symbolic Logic 72 (2007), 1379-1384; doi:10.2178/js1/1203350793. 
[21] D.S. Bridges and F. Richman, Varieties of Constructive Mathematics, Cambridge University Press, 1987.

[22] D.S. Bridges, F. Richman, and P. Schuster, Linear independence without choice, Ann. Pure Appl. Logic 101 (2000), 95-102; doi:10.1016/S0168-0072(99)00030-5.

[23] D.S. Bridges, F. Richman, and P. Schuster, A weak countable choice principle, Proc. Amer. Math. Soc. 128 (2000), 2749-2752.

[24] D.S. Bridges, and L. Vîţă, Techniques of Constructive Analysis, Springer, New York, 2006.

[25] H. Diener and I. Loeb, Sequences of real functions on $[0,1]$ in constructive reverse mathematics, Ann. Pure Appl. Logic 157 (2009), 50-61; doi:10.1016/j.apal.2008.09.018.

[26] H. Diener and P. Schuster, Uniqueness, Continuity, and Existence of Implicit Functions in Constructive Analysis, in: A. Bauer, P. Hertling, Ker-I Ko, eds., 6th Int'l Conf. on Computability and Complexity in Analysis 2009, Schloss Dagstuhl-Leibniz-Zentrum für Informatik, Dagstuhl, 2009. http://drops.dagstuhl.de/opus/volltexte/2009/2265. Also: LMS J. Comput. Math., to appear.

[27] H.M. Friedman, Set Theoretic Foundations for Constructive Analysis, Ann. Math. 105 (1977) 1-28; doi:10.2307/1971023.

[28] H. Ishihara, An omniscience principle, the König lemma and the Hahn-Banach theorem, Z. Math. Logik Grundlag. Math. 36 (1990), 237-240; doi:10.1002/malq.19900360307.

[29] H. Ishihara, Informal constructive reverse mathematics, Sūrikaisekikenkyūsho Kōkyūroku 1381 (2004), 108-117.

[30] H. Ishihara, Constructive reverse mathematics: compactness properties, From Sets and Types to Topology and Analysis, Oxford Logic Guides 48, Oxford University Press (2005), 245-267; doi:10.1093/acprof:oso/9780198566519.003.0016.

[31] H. Ishihara, Reverse mathematics in Bishop's constructive mathematics, Philosophia Scientiae, cahier spécial 6 (2006), 43-59.

[32] H. Ishihara, Weak König's Lemma implies Brouwer's Fan Theorem: a direct proof, Notre Dame J. Formal Logic 47 (2006), 249-252; doi:10.1305/ndjfl/1153858649.

[33] H. Ishihara, Unique existence and computability in constructive reverse mathematics, Computation and Logic in the Real World, Third Conference on Computability in Europe, CiE 2007. Siena, Italy, June 2007. Springer, Berlin and Heidelberg. Lect. Notes Comput. Sci. 4497 (2007), 368-377; doi:10.1007/978-3-540-73001-9_38.

[34] U. Kohlenbach, Effective moduli from ineffective uniqueness proofs: An unwinding of de La Vallée Poussin's proof for Chebycheff approximation, Ann. Pure Appl. Logic 64 (1993), 27-94; doi:10.1016/0168-0072(93)90213-W.

[35] U. Kohlenbach, and P. Oliva, Proof mining: a systematic way of analysing proofs in mathematics, Proc. Steklov Inst. Math. (2003), no. 3 (242), 136-164. 
[36] U. Kohlenbach, Applied Proof Theory: Proof Interpretations and their Use in Mathematics, Springer, Berlin and Heidelberg, 2008.

[37] KreĬnovič, V.Ja., Review of 'Constructive Functional Analysis', MR0521982 (82k:03094).

[38] V.A. Lifshitz, Investigation of constructive functions by the method of fillings, J. Soviet Math. 1 (1973), 41-47; doi:10.1007/BF01117468.

[39] I. Loeb, Equivalents of the (weak) fan theorem, Ann. Pure Appl. Logic 132 (2005), 51-66; doi:10.1016/j.apal.2004.07.002.

[40] I. Loeb, Indecomposability of $\mathbb{R}$ and $\mathbb{R} \backslash\{0\}$ in constructive reverse mathematics, Log. J. IGPL 16 (2008), 269-273; doi:10.1093/jigpal/jzn006.

[41] I. Loeb, Indecomposability of negative dense subsets of $\mathbb{R}$ in constructive reverse mathematics, Log. J. IGPL 17 (2009), 173-177; doi:10.1093/jigpal/jzp002.

[42] J. Myhill, Constructive set theory, J. Symbolic Logic 40 (1975), 347-382; doi: $10.2307 / 2272159$.

[43] F. Richman, Intuitionism as generalization, Philos. Math. (3)5 (1990), 124-128.

[44] F. Richman, The fundamental theorem of algebra: a constructive development without choice, Pacific J. Math.196 (2000), 213-230; doi:10.2140/pjm.2000.196.213.

[45] F. Richman, Spreads and choice in constructive mathematics, Indag. Math. (N.S.) 13 (2002), 259-267; doi:10.1016/S0019-3577(02)80009-X.

[46] P. Schuster, Unique existence, approximate solutions, and countable choice, Theoret. Comput. Sci. 305 (2003), 433-455; doi:10.1016/S0304-3975(02)00707-7.

[47] P. Schuster, Countable choice as a questionable uniformity principle, Philos. Math. (3) 12 (2004), 106-134.

[48] P. Schuster, What is continuity, constructively? J. UCS 11 (2005), 2076-2085.

[49] P. Schuster, Unique solutions, Math. Log. Quart. 52 (2006), 534-539; doi:10.1002/malq.200610012. Corrigendum: Math. Log. Quart. 53 (2007), 214.

[50] P. Schuster, Problems, solutions, and completions, J. Logic Algebr. Program. 79 (2010), 84-91; doi:10.1016/j.jlap.2009.02.007.

[51] P. Schuster, and H. Schwichtenberg, Constructive solutions of continuous equations, One Hundred Years of Russell's Paradox. International Conference in Logic and Philosophy. München, Germany, June 2001. De Gruyter, Berlin De Gruyter Series in Logic and Its Applications 6 (2004), 227-245.

[52] H. Schwichtenberg, A direct proof of the equivalence between Brouwer's fan theorem and König's lemma with a uniqueness hypothesis, J. UCS 11 (2005), 2086-2095.

[53] P. Taylor, A lambda calculus for real analysis, J. Log. Anal. 2 (2010), Paper 5, 115 pp.; doi:10.4115/jla.2010.2.5.

[54] W. Veldman, Brouwer's fan theorem as an axiom and as a contrast to Kleene's alternative, Preprint, Radboud University, Nijmegen, 2005. 
[55] W. Veldman, Brouwer's approximate fixed-point theorem is equivalent to Brouwer's fan theorem, in: S. Lindström, E. Palmgren, K. Segerberg, V. Stoltenberg-Hansen, eds., Logicism, Intuitionism, and Formalism-What has become of them? Workshop on the Philosophy of Mathematics, Uppsala, Sweden, August 27-29, 2004. Springer, Dordrecht. Synth. Libr. 341 (2009), 277-299; doi:10.1007/978-1-4020-8926-8_14.

[56] K. Weihrauch, Computable Analysis. An Introduction. Springer, Berlin, 2000; doi:10.1007/11494645_64.

Department of Pure Mathematics, University of Leeds, Leeds LS2 9JT, England

Department of Pure Mathematics, University of Leeds, Leeds LS2 9JT, England

mmmrh@leeds.ac.uk, pschust@maths.leeds.ac.uk

Received: 3 May 2010 Revised: 21 December 2010 